\title{
AN INTELLIGENT KNOWLEDGE-BASED RECOMMENDATION SYSTEM
}

\author{
Xiaowei Shi \\ Philips Shanghai R\&D Centre, P.R.C.,Email:nancy.shi@philips.com
}

\begin{abstract}
An intelligent knowledge-based recommendation system for multiple users in TV-Anytime environment is developed. The architecture of the multi-agent recommendation system is described. KQC (Keyword-query combination) user profile is proposed for information modeling. The priority filtering agent operates according to the similarity between the program and the KQC user profile. This knowledge-based recommendation system can provide personalized content to users based on their preferences more efficiently and more concisely.
\end{abstract}

Key words: Recommendation, User profile, Metadata, Knowledge Representation, Multiagent

\section{INTRODUCTION}

With the rapid growth of communication technologies, there is an overabundance of DTV programs available. This precipitates a need for smart "recommender" to help people obtain personalized programs.

Existing recommendation system such as TV-Advisor and Personal TV $[1,2,3]$ are based on basic EPG (Electronic Program Guide) metadata which includes program title, start time, and duration for each channel.

Today, metadata includes more detailed information such as genre, actors, directors, and so on. This provides a potential possibility for users to navigate and search the content more easily. In order to achieve this purpose, the metadata need to be standardized. The TV-Anytime Forum is working hard to set this standard [5]. 
In order to recommend the contents needed by users more efficiently, this paper proposes an intelligent program recommendation system based on KQC user profile and priority filtering method in the TV-Anytime environment.

\section{INTELLIGENT INFORMATION MODELLING}

To minimize the requirements for memory and processing, a lightweight KQC(Keyword-query combination) user profile is proposed for user modeling. The KQC user profile consists of two parts: 1) instant queries from users. The queries can be both explicit and implicit, such as a specific program or some specific types of information which user wants to look; 2) usual interest and dislike features. The following describes the structure of user profile in detail.

i> Part 1: instant query

Instant queries can be either explicit or implicit, such as a specific program or some specific types of information which user wants to look. Its priority is very high; but it will be updated for the next time. The part 1 is represented as:

$$
P_{1}=\left(\left(t_{1}, c_{1}\right),\left(t_{2}, c_{2}\right), \ldots,\left(t_{m}, c_{m}\right)\right)
$$

Where $t_{i}$ represent a feature and $c_{i}$ means the content of a feature $t_{i}$.

ii $>$ Part 2: usual preference

For the usual interest and dislike features, to minimize the requirement for memory and processing, each feature is defined as a three-dimension vector (feature, weight, like-degree). If there are $m$ different features in Part a of a profile, it is represented as:

$$
P_{2}=\left(\left(t_{1}, w_{1}, l d_{1}\right),\left(t_{2}, w_{2}, l d_{2}\right), \ldots,\left(t_{m}, w_{m}, l d_{m}\right)\right)
$$

where $t_{i}$ is a feature; $w_{i}$ is the weight of feature $t_{i} ; l d_{i}$ is the like-degree of feature $t_{i}$.

\section{MULTI-AGENT RECOMMENDATION SYSTEM}

\subsection{System Architecture}

The recommendation system developed in this paper is based on multiagent. Its structure is shown in Figure 1. The whole system includes three agents: the Priority Filtering Agent, the Adaptation Agent, and the User Agent. The User Agent consists of two subagents: the Feedback Report Agent and the Interactive Agent. 


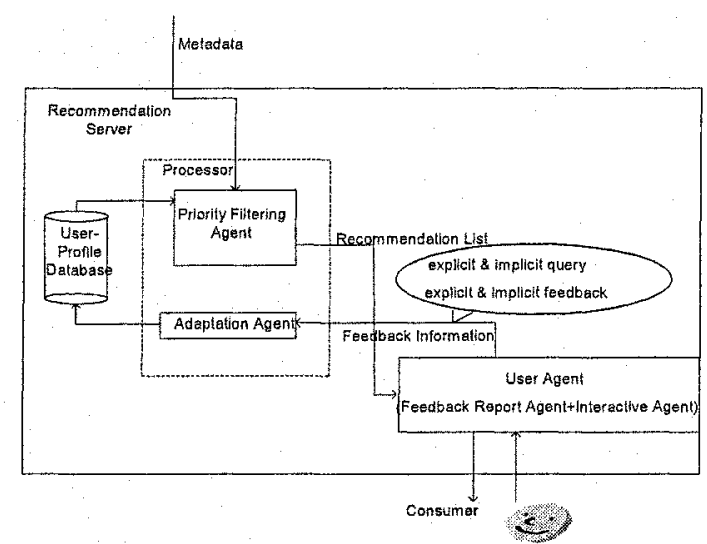

Figure 1. System architecture

The multi-agent recommendation system is flexible. It is easier to be modulated such as adding new agents for more function, or being divided into two parts (third party side, user side) to reduce the cost of the user side.

\subsection{Priority Filtering Agent}

The Priority Filtering Agent filters the information according to the similarity between the program and the user. Figure 2 shows the systematic framework of the Filtering Agent.

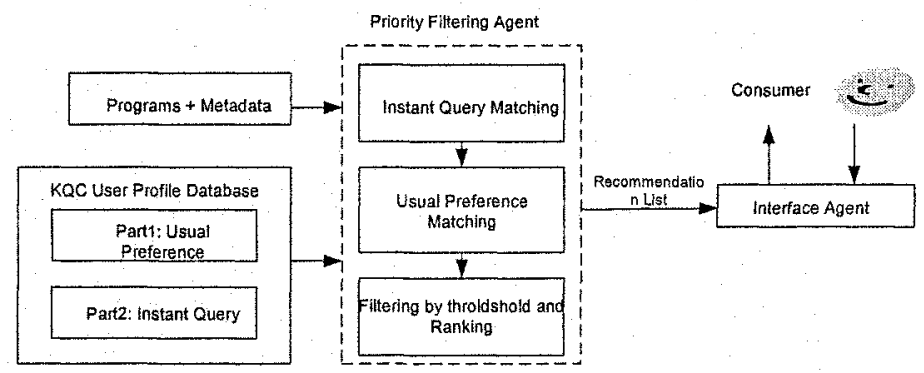

Figure 2. Filtering Agent systematic framework

When a piece of information metadata arrives, the Filtering Agent will evaluate it according to the priority. For each user, the recommended content is classified into two classes: content matching instant query and content matching usual interest. Different ways are used to deal with different part of the user profile (instant query, usual interest).

For matching instant query, if a query is a specific title, the Priority Filtering Agent directly select the content named by the title; If a query is a 
kinds of program, the Filtering Agent select the content that has the same properties.

For usual interests and dislikes, the similarity between the information $\mathrm{C}$ and profile $\mathrm{P} 2$ is calculated by:

$$
\operatorname{sim}\left(C, P_{2}\right)=\frac{C \times P_{2}}{\|C\| \times\left\|P_{2}\right\|}
$$

If the calculated similarity is above the preset threshold $\theta$, the program is considered to be relevant to the user's preference.

Finally the Priority Filtering Agent provides a recommendation list to the user according to the ranking of the information. The recommended information that meets the user's querying has the highest priority. Others are ranked based on their similarities.

\subsection{User Agent}

The User Agent is Friendly and Secure. It consists of the Feedback Report Agent and the Interactive Agent. The Feedback Report Agent will report the program's interest degree to the Interactive Agent. The interest degree represents the user's viewing behavior.

The Interactive Agent provides the users a chance to modify the feedback report. In addition, the users can add their new interest, new need in this step. After that, feedback information is sent to the Adaptation Agent.

\subsection{Adaptation Agent}

The Adaptation Agent revises the user's profile based on feedback information. For the user queries, the Adaptation Agent will put it in Part 1 (instant query) of the user profile directly and clear up the old Part 1. For other feedback information, the updating process only updates the features related to the content that the user has very different feeling from the similarity evaluation.

\section{APPLICATION SCENARIO}

An application scenario is provided. Figure 3 shows the main user interface of the recommendation system. Functions are listed in the left column. A program recommendation list is on the topside of middle column. The description of a selected program is presented in the bottom of middle column. The interface contains a media player. For a selected program, three choices are provided to the user, which are "Display", "Delete", and "Skip". 


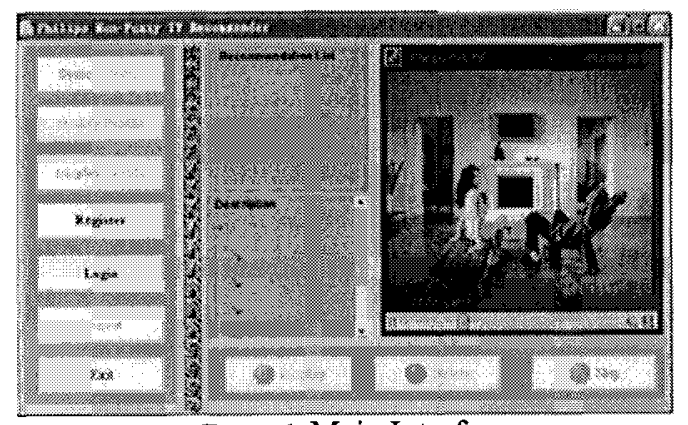

Figure 1. Main Interface

\section{CONCLUSION}

An intelligent multi-agent knowledge-based recommendation system is developed to provide personalized content for multiple users in TV-Anytime environment. The proposed KQC user profile can express the user's need clearly. During filtering, ranking and updating processes, the instant query part of the user profile has higher priority than the usual interest part, so the processes computation cost is reduced and the more reasonable recommendation list is gotten. The recommendation systems can be equipped in PDR or STB. From the application scenario, a friendly user interface is provided to help the user enjoy life more freely.

\section{REFERENCES}

1. Belkin, N.J. \& Croft, W.B.. "Information filtering and information retrieval: two sides of the same coin". Communications of the ACM, 1994

2. P. W. Foltz and S. T. Dumais. "Personalized information delivery: An analysis of information filtering methods". Communications of the ACM, 1992

3. P W Foltz and S T Dumais. "Personalized information delivery: An analysis of information filtering methods". Communications of the ACM, 1992

4. Zheng Zhikai, Zhang Guangfan and Shao Huihe. Data Mining and Knowledge Discovery: An Overview and Prospect. Information and Control, 1999, 28(5): 357-365.

5. TV-Anytime Metadata Specifications Document, SP003v12 Part A AppendixB, TVAnytime Forum, Jun. 2002. 